\title{
Genotype-phenotype Correlation in Pelizaeus Merzbacher Disease and Pelizaeus Merzbacher-like Disease
}

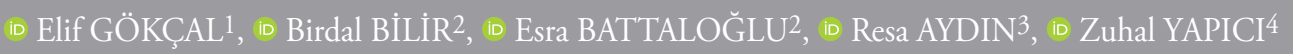

${ }^{1}$ Bezmialem Vakıf University Faculty of Medicine Hospital, Deparment of Norology, İstanbul, Turkey

${ }^{2}$ Boğaziçi University Faculty of Science, Department of Molecular Biology and Genetics, İstanbul, Turkey

3istanbul University İstanbul Faculty of Medicine, Department of Physical Therapy and Rehabilitation, İstanbul, Turkey

4Istanbul University İstanbul Faculty of Medicine, Department of Neurology, İstanbul, Turkey

\begin{abstract}
Objective: Among the hypomyelinating diseases of childhood, Pelizaeus Merzbacher disease (PMD) is caused by X-linked proteolipid protein (PLP) gene mutations, whereas patients without mutations of PLP gene-called Pelizaues Merzbacher-like disease (PMLD) have recessive gap junction protein $\alpha 12$ (gap junction alpha-12/gap junction gamma-2) gene mutations. The aim of this study was to evaluate clinical severity and progression in time in patients with PMD and PMLD.

Methods: The motor developmental stages of the patients were reviewed; disease severity was classified according to the walking ability they were able to achieve. Progression pattern was determined according to comparison of neurological findings at the time of the study and at follow-up visits. Patients with PMD and PMLD were compared in terms of disease severity and progression rates as well as patient groups with a unique causative mutation were analyzed individually.

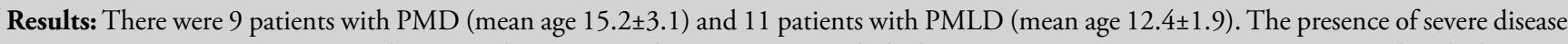
was more common in patients with PMD when compared to PMLD. In X-linked PMD, missense mutations were associated with the most severe disease and rapid progression, while deletion mutations were associated with mild disease severity and slow progression. Disease severity and progression patterns seemed to be heterogenous in different causative mutations of PMLD.

Conclusion: Although PMLD might have milder disease phenotype when compared to PMD, certain causative mutations in different genetic traits may cause different disease severity and progression patterns.
\end{abstract}

Keywords: Pelizaues-Merzbacher disease, Pelizaues-Merzbacher-like Disease, gap junction protein $\alpha 12$, genotype, phenotype

\section{Introduction}

Pelizaeus-Merzbacher disease (PMD) is the prototype of hypomyelinating diseases of childhood which was firstly described as an X-linked disorder caused by mutations or rearrangements in the proteolipid protein 1 (PLP1) gene. Causative mutations of the PLP1 gene are-in declining order of frequency-duplications, point mutations, insertions, and deletions (1). Clinical features include nystagmus (typically occurs in the first months of life and resolves within 2-5 years of age), dysarthria, ataxia, hypotonia and developmental delay evolving into spastic quadriplegia in the first years of life (2). The clinical severity and rate of progression vary widely, probably depending on the variability of the causative mutation (1). Although, it was firstly described as a X-linked disorder, patients with PMD phenotype but without mutations of PLP1 gene were shown to have an autosomal recessive (OR) trait. One of the causative mutations in these patients -called
Address for Correspondence/Yazışma Adresi: Elif GÖKÇAL, Bezmialem Vakıf University Faculty of Medicine Hospital, Department of Neurology, İstanbul, Turkey

Phone: +90 2124531700 E-mail: elifdr99@gmail.com ORCID ID: orcid.org/0000-0003-3309-4368
Received/Geliş Tarihi: 05.10 .2018 Accepted/Kabul Tarihi: 17.10 .2018

Cite this article as: Gökçal E, Bilir B, Battaloğlu E, Aydın R, Yapıcı Z. Genotype-phenotype Correlation in Pelizaeus Merzbacher Disease and Pelizaeus Merzbacher-like Disease. Bezmialem Science 2019;7(3):215-20 
Pelizaeus-Merzbacher-like disease (PMLD)- is in gap junction protein $\alpha 12$ gene/ gap junction gamma (GJA12/GJC2) coding for Connexin 47 (3). Although GJA12/GJC12 mutations are found in a minority of PMLD, its frequency is higher in Turkish patients (4). The data about which phenotypical differences occur in these different types of genetic traits are limited. In this study, we aimed to compare the age of onset, clinical severity and progression rates in PMD and PMLD.

\section{Methods}

The data of this study were obtained from 20 patients who had been followed between 1995-2011 in the İstanbul Medical Faculty, Department of Neurology. All genetic analysis of patients and families were performed in the Department of Molecular Biology and Genetics in Bogaiçi University. Only patients who had a causative mutation were included into the study. Screening tests for metabolic diseases were performed. All patients underwent brain magnetic resonance imaging (MRI). Exclusion criteria were as following: a) the presence of any other congenital, metabolic or developmental brain diseases, b) the absence of follow-up visits, c) rejection to give written informed consent for the study.

The history of birth and motor developmental stages of the patients were learned from parents as well as from clinical charts. Detailed neurological examination was performed in all patients and compared with the clinical findings at follow-up visits. Moreover, functional independence measurement (FIM/ weeFIM), scale for the assessment and rating of ataxia (SARA) and Modified Achworth Spasticity scale (MAS) were also evaluated in all patients. FIM is an 18-item motor and cognitive function test that is used to assess a patient's level of functionality (5). weeFIM is, on the other hand, used to assess this functional independence in children (6). The total score for the FIM/weeFIM instrument (the sum of the motor and cognition subscale scores) will be a value between 18 and 126 . The higher the score, the more independent the patient is according to the FIM/weeFIM scores. SARA is a clinical scale to assess ataxia (7). It has 8 categories with accumulative score ranging from 0 (no ataxia) to 40 (most severe ataxia). The MAS is the most widely used clinical scale to measure the increase of muscle tone (8). It has 5 categories ranging between 0 (no increase in muscle tone) and 4 (affected parts rigid in extension or flexion). In the study, the MAS score was scored according to the tone of lower extremities.

According to the obtained data, the patients were grouped according to the development of walking ability as follows: a) group 1 (severe disease) included patients who never had the ability to walk, b) group 2 (moderate disease) included patients who had the ability to walk with assistance, c) group 3 (mild disease) included patients who had the ability to walk without assistance during the developmental stages. Groups were compared in terms of age, sex, clinical scores, genetic traits and causative mutations. progression-which was accepted if the patient lost the major activities that he/she was able to do (walking, sitting and speech) during the follow-up- was evaluated in every patient. Categorical variables were presented as percentage (\%) and continuous variables as mean $\pm S D$ or median [interquartile range, (IQR)], as appropriate. The study approved by İstanbul University, İstanbul Medical Faculty Ethics Comittee (2011/807-567).

\section{Statistical Analysis}

Categorical variables were analyzed using pearson chi-square or Fisher's Exact test, and continuous variables using the independent-sample t-test (for normal distributions) and Kruskal-Wallis test (for non-normal distributions). Statistical assessments were performed using SPSS software pack (Statistical Package for Social Sciences for Windows Version 23 software). A p value of less than 0.05 was considered statistically significant.

\section{Results}

A total of 20 patients (14 female, 6 male) with a mean age of 13.7 \pm 7.84 years (minimum-maximum: 4-30) were included in the study. Follow-up period was 3-12 years. According to the genetic analysis, 9 patients had X-linked PMD and the remaining 11 patients had OR PMLD trait. The mean age between X-linked and OR patients was not different $(15.2 \pm 3.1$ vs 12.4 $\pm 1.9, \mathrm{p}=0.448)$. Detailed information about demographics, causative mutation and developmental stages according to the walking ability are given in Table 1 .

There was no difference between 3 groups in terms of age $(\mathrm{p}=0.905)$, FIM/weeFIM score $(\mathrm{p}=0.085)$, SARA score $(\mathrm{p}=0.313)$ and MAS ( $\mathrm{p}=0.305)$. Of patients with X-linked PMD, 33.3\% were in the severe, $33.3 \%$ in moderate and the remaining $33.3 \%$ were in mild disease group. Of PMLD, $18.2 \%$ were in the severe, $45.2 \%$ in moderate and the remaining $36.4 \%$ were in mild disease group.

In 5 patients with a duplication in PLP1 gene, 3 patients never had the ability to walk, 2 patients, other hand, had at once the ability to walk with assistance. Of these patients, case 5 ( 29 years of age) was still able to walk with assistance and he had only mild ataxia. When compared with the neurological examination performed 5 years before, he had a very slow progression. The other patient in moderate disease group was patient 1 who was 7 years of age. According to the follow-up visits, this patient had lost the walking ability with a progression in ataxia. The 2 patients with comparative genomic hybridization duplication had also apparently progressed during follow-up period. The 2 patients with GJA12/GJC2 duplications had the ability to walk with assistance, but both lost this ability during the 8-year follow-up period. Patient 7 (17 years of age) with a deletion in PLP1 gene had the ability to walk but it was late according to normal motor developmental stages of childhood. Progression of the disease was slow in early follow-up period, but he was not able to walk at the time of study. Of 4 patients with a deletion in GJA12/GJC2 gene, two patients never had the ability to walk, the other two patients, on the other hand, had the ability to walk 
Table 1. Demographics, genetic characteristics and motor development stages of all patients

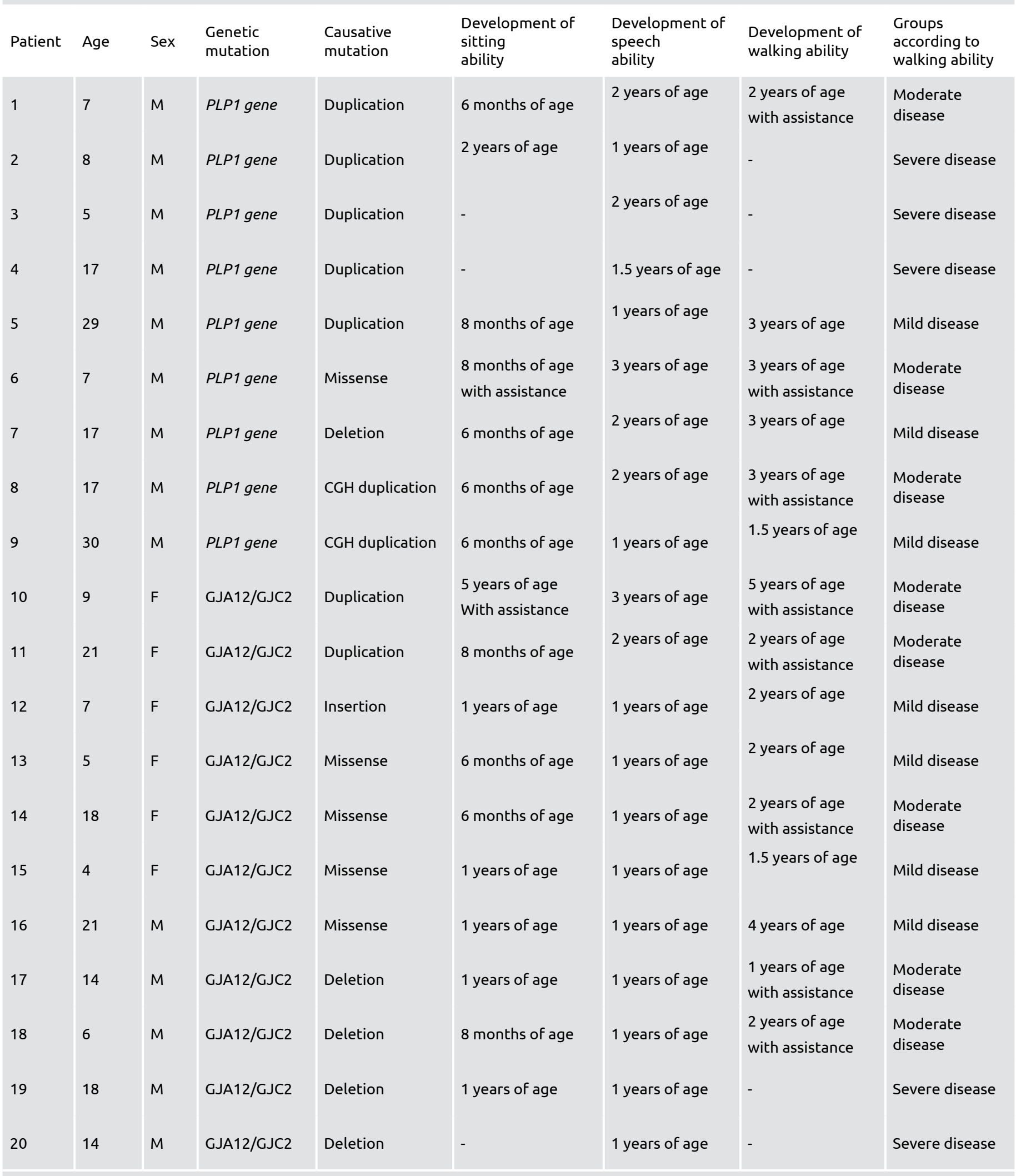

PLP: Proteolipid protein, GJA12: Gap junction alpha12, GJC2: Gap junction gamma2, CGH: Comporotive genomic hybridizotion 
Table 2. Comparison of clinical scales between patients with PMD and PMLD

\begin{tabular}{|c|c|c|c|}
\hline Clinical scales & PMD & PMLD & $p$ \\
\hline SARA, median (IQR) & $35(29.5-39)$ & $37(15-40)$ & 0.788 \\
\hline Ashworth spasticity score, median (IQR) & $2(1.5-3.5)$ & $3(0-4)$ & 0.816 \\
\hline
\end{tabular}

with assistance, one of whom (6 years of age) was still able to walk at the time of study. The patient 6 (7 years of age) with a missense mutation in PLP1 gene had the ability to walk with assistance however he lost this ability in a short time period. All of 4 patients with missense mutation in GJA12/GJC2 gene had the ability to walk with assistance. Of these, 2 patients who were in the age of 18 and 21 lost this ability during the recent 5 -year follow-up period. The only patient with an insertion in GJA12/ GJC2 gene (patient 12; 7 years of age) had completed motor developmental stages in time. He was able to walk without assistance at the time of the study.

Regarding to clinical scales, there were no difference in terms of FIM/weeFIM, SARA and MAS scores between PMD and PMLD (Table 2). Age did not correlate in any of these scales.

\section{Discussion}

To our knowledge, this study has the largest series of Turkish patients and evaluates the association of phenotypic characteristics in the patients with PMD and PMLD. Both PMD and PMLD are hypomyelinating disorders with similar clinical and neuroradiological phenotypes. The patients present in infancy with nystagmus, jerky head movements, hypotonia, ataxia and developmental delay evolving into spastic paraplegia (9).

There have been numerous genetical and molecular studies that investigate the nature of the PMD and other hypomyelinating disorders (10-12). However, data about the clinical features and prognosis of the patients with genetically confirmed PMD and PMLD are limited. In a study in which types in patients having point mutations in PLP1 gene were investigated, clinical severity was found to be correlated with the nature of the mutation. Single amino-acid mutations in highly-conserved region of PLP caused the most severe forms of PMD (13). Another study that investigated the phenotype-genotype correlation in 5 patients with PMD with duplication mutation, did not find any correlation with the extent of the duplicated genomic segments and the clinical severity (14). In a previous report in our patient population carrying duplication mutation (4), the severity of the disease did not show any correlation with the duplication size in contrary to previously reported studies $(15,16)$. Our study did not focus on genetic basis of the disease, instead we compared clinical features and prognosis in two different traits and mutations in PMD and PMLD. Although PMD is known as the prototype of hypomyelinating diseases, PMLD caused by OR genetic trait has been reported to be seen in high frequency in Turkey as well as in other Eastern countries, probably depending on high rate of consanguineous marriages $(4,17,18)$.

In a study that investigated clinical features of 16 patients with PMLD according to developmental scores, the majority of the patients were found to have ambulation capacities, speech and good cognitive functions (3). Our results also support that PMLD has better clinical severity and prognosis when compared to X-linked PMD. When we analyzed different types of causative mutations in all patients, patients with X-linked duplications constituted the most heterogenous patient group in terms of clinical severity and progression. OR duplications, on the other hand, had a moderate clinical severity and progression was not severe and these patients had milder ataxia and hypotonia even in late stages of the disease. In patients with X-linked deletion mutation, clinical findings were milder and progression rate was slower compared with other causative mutations, similar to previous publications (19). Clinical findings both in early and late stages of the disease were severe in patients with $\mathrm{X}$-linked missense mutations, as previously reported (13). On the other hand, OR missense mutations had moderate severity and the progression was slower. The only patient with insertion in GJA12/ CJG2 had moderate severity and slow progression rate. We did not find any differences in terms of clinical scores between PMD and PMLD, probable due to the small size of the cohort as well as the absence of age-matching PMD and PMLD patient groups. Although the mean age between PMD and PMLD was not different, we used two different scale formats according to the age (FIM vs weeFIM) for functional independency. Ataxia rating scales are reported to be age-dependent even in healthy children (20). Nevertheless, clinical scales enhance the communication between health professionals and they increase the objectivity when used to compare the patients with similar clinical findings.

There are limitations of the study. Despite we performed detailed neurological examinations and interviews with the families to identify the severity of the disease, the evaluation of progression was based on the retrospective review of clinical charts. Moreover, clinical scales we used at the time of present study were not be performed at the previous follow-up visits. Previous studies reported similar neuroradiological findings in patients with PMD and PMLD (21). We did not report MRI findings in patient groups, however MRI results were very similar in our patient series based on an unpublished data. 
In a study comparing neurophysiologic findings between PMD and PMLD reported a clear difference in brain stem evoked potentials (BAEP) (22). However, BAEP studies were not performed in our patients.

\section{Conclusions}

With the increase in the use of MRI as well as the availability of genetical analysis, the number of patients receiving a diagnosis of PMD and PMLD have been increasing. However, the similarities in clinical features between them may complicate the decision of the appropriate genetic testing particularly if there is no evidence of genetic transformation pattern based on patient's pedigree. It might be helpful to know that X-linked PMD patients may have more severe disease when compared to OR PMLD patients. Certain causative mutations in different genetic traits differ in terms of clinical severity and progression rates. These data can be used when informing the family members about the possible course of the disease according to the existing causative mutation. Further prospective studies are needed in larger patient series.

\section{Ethics}

Ethics Committee Approval: The study approved by İstanbul University, İstanbul Medical Faculty Ethical Comittee (2011/807-567) .

Informed Consent: Written informed consent was obtained from all participants and/or their parents.

Peer-review: Externally peer-reviewed.

\section{Authorship Contributions}

Concept: Z.Y., R.A., Design: E.G., R.A., Z.Y., Data Collection or Processing: E.G., Z.Y., Analysis or Interpretation: E.G., R.A., B.B., E.B., Literature Search: E.G., Z.Y., Writing: E.G., Z.Y.

Conflict of Interest: No conflict of interest was declared by the authors.

Financial Disclosure: The authors declared that this study received no financial support.

\section{References}

1. Koeppen AH, Robitaille Y. Pelizaeus-Merzbacher disease. J Neuropathol Exp Neurol 2002;61:747-59.

2. Boulloche J, Aicardi J. Pelizaeus-Merzbacher disease: clinical and nosological study. J Child Neurol 1986;1:233-9.

3. Henneke M, Combes P, Diekmann S, Bertini E, Brockmann K, Burlina AP. GJA12 mutations are a rare cause of PelizaeusMerzbacher-like disease. Neurology 2008;70:748-54.

4. Bilir B, Yapici Z, Yalcinkaya C, Baris I, Carvalho CM, Bartnik M, et al. High frequency of GJA12/GJC2 mutations in Turkish patients with Pelizaeus-Merzbacher disease. Clin Genet 2013;83:66-72.

5. Linacre JM, Heinemann AW, Wright BD, Granger CV, Hamilton BB. The structure and stability of the Functional Independence Measure. Arch Phys Med Rehabil 1994;75:127-32.
6. Msall ME, DiGaudio K, Duffy LC, LaForest S, Braun S, Granger CV. WeeFIM: Normative Sample of an Instrument for Tracking Functional Independence in Children. Clin Pediatr (Phila) 1994;33:431-8.

7. Schmitz-Hübsch T, Du Montcel ST, Baliko L, Berciano J, Boesch S, Depondt C, et al. Scale for the assessment and rating of ataxia: development of a new clinical scale. Neurology 2006;66:1717-20.

8. Meseguer-Henarejos AB, Sánchez-Meca J, López-Pina JA, CarlesHernández R. Inter- and intra-rater reliability of the Modified Ashworth Scale: a systematic review and meta-analysis. Eur J Phys Rehabil Med 2018;54:576-90.

9. Hobson GM, Garbern JY. Pelizaeus-Merzbacher disease, PelizaeusMerzbacher-like disease 1, and related hypomyelinating disorders. Semin Neurol 2012;32:62-7.

10. Southwood CM, Garbern J, Jiang W, Gow A. The unfolded protein response modulates disease severity in pelizaeus-merzbacher disease. Neuron 2002;36:585-96.

11. Gow A, Southwood CM, Lazzarini RA. Disrupted proteolipid protein trafficking results in oligodendrocyte apoptosis in an animal model of pelizaeus-merzbacher disease. J Cell Biol 1998;140:925-34.

12. Mimault C, Giraud G, Courtois V, Cailloux F, Boire JY, Dastugue $\mathrm{B}$, et al. Proteolipoprotein gene analysis in 82 patients with sporadic pelizaeus-merzbacher disease: duplications, the major cause of the disease, originate more frequently in male germ cells, but point mutations do not. Am J Hum Genet 1999;65:360-9.

13. Cailloux F, Gauthier-Barichard F, Mimault C, Isabelle V, Courtois V, Giraud G, et al. Genotype-phenotype correlation in inherited brain myelination defects due to proteolipid protein gene mutations. Clinical European Network on Brain Dysmyelinating Disease. Eur J Hum Genet 2000;8:837-45.

14. Regis S, Biancheri R, Bertini E, Burlina A, Lualdi S, Bianco MG, et al. Genotype-phenotype correlation in five Pelizaeus-Merzbacher disease patients with PLP1 gene duplications. Clin Genet 2008;73:279-87.

15. Inoue K, Osaka H, Imaizumi K, Nezu A, Takanashi J-I, Arii J, et al. Proteolipid protein gene duplications causing Pelizaeus-Merzbacher disease: Molecular mechanism and phenotypic manifestations. Ann Neurol 1999;45:624-32.

16. Woodward K, Kendall E, Vetrie D, Malcolm S. Pelizaeus-Merzbacher disease: identification of $\mathrm{Xq} 22$ proteolipid-protein duplications and characterization of breakpoints by interphase FISH. Am J Hum Genet 1998;63:207-17.

17. Bugiani M, Al Shahwan S, Lamantea E, Bizzi A, Bakhsh E, Moroni I, et al. GJA12 mutations in children with recessive hypomyelinating leukoencephalopathy. Neurology 2006;67:273-9.

18. Karimzadeh P, Ahmadabadi F, Aryani O, Houshmand M, Khatami A. New Mutation of Pelizaeus-Merzbacher-Like Disease; A Report from Iran. Iran J Radiol 2014;11:e6913.

19. Nevin ZS, Factor DC, Karl RT, Douvaras P, Laukka J, Windrem MS, et al. Modeling the Mutational and Phenotypic Landscapes of Pelizaeus-Merzbacher Disease with Human iPSC-Derived Oligodendrocytes. Am J Hum Genet 2017;100:617-34.

20. Brandsma R, Spits AH, Kuiper MJ, Lunsing RJ, Burger H, Kremer HP, et al. Ataxia rating scales are age-dependent in healthy children. Dev Med Child Neurol 2014;56:556-63. 
21. Steenweg ME, Vanderver A, Blaser S, Bizzi A, de Koning TJ, Mancini $\mathrm{GM}$, et al. Magnetic resonance imaging pattern recognition in hypomyelinating disorders. Brain 2010;133:2971-82.
22. Henneke M, Gegner S, Hahn A, Plecko-Startinig B, Weschke B, Gärtner $\mathrm{J}$, et al. Clinical neurophysiology in GJA12-related hypomyelination vs Pelizaeus-Merzbacher disease. Neurology 2010;74:1785-9. 